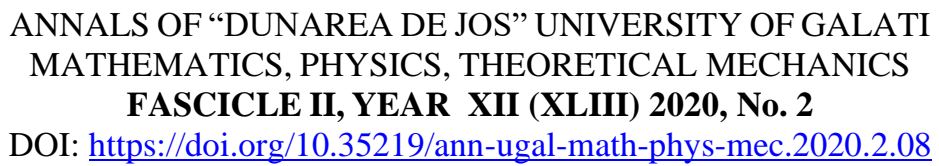

\title{
Proving the environmental impact of NBOMe clandestine laboratories: a new analytical tool
}

\author{
Adelina Ion ${ }^{1}$, Steluta Gosav ${ }^{1,2}$, Mirela Praisler ${ }^{1,2}$ \\ 1 "Dunarea de Jos" University of Galati, Faculty of Sciences and Environment, 47 Domneasca \\ Street, 800008, Galati, Romania, \\ Adelina.Ion@ugal.ro,sgosav@ugal.ro,Mirela.Praisler@ugal.ro \\ ${ }^{2}$ INPOLDE interdisciplinary research network, Dunarea de Jos University of Galati, 111 Domneasca St., \\ 800201 Galati, Romania
}

\begin{abstract}
The research performed during the last decades has allowed a better understanding not only of the moral but also of the environmental implications of drug abuse. It has indicated that the traditional approaches for reducing drug abuse are no longer enough. New and more efficient analytical tools are needed for the detection of clandestine laboratories and for bringing to courts of law irrefutable evidence for their environmental impact.

NBOMes are a family of synthetic psychotropic drugs that have increasingly been detected in seizures made by law enforcement all around the world. This paper presents a new analytical tool that can be used for detecting not only these substances of abuse but also the main precursors that are used for their manufacture.
\end{abstract}

Keywords: NBOMe hallucinogens, molecular descriptors, ANN.

\section{INTRODUCTION}

A recently detected class of strong synthetic psychotropic drugs are dimethoxyphenyl-N-[(2methoxyphenyl) methyl] ethanolamine (NBOMes) derivatives, initially developed for research purposes [1]. 25I-NBOMe (IUPAC name: 2- (4-iodo-2,5-dimethoxyphenyl) -N - [(2-methoxyphenyl) methyl] ethanamine) is a derivative of 2,5-dimethoxy-4-iodophenethylamine (2C-I), which is often sold through illicit channels in various forms, such as blotter paper, liquids, powders, or capsules [2]. This potent illicit hallucinogen, whose serotonergic action is similar to that of LSD or psilocin, is sold under the names N-bomb, N-bome, Smiles, 25I, Bom-25, or Cimbi-5 (see Fig. 1). Consumption of 25INBOMe has negative effects on human health, such as visual and auditory hallucinations, confusion, agitation, anxiety, vasoconstriction, increased heart rate, thought loops, nausea, mydriasis, increased blood pressure, acute kidney injury, oxygen desaturation. According to recent clinical toxicology studies, this compound has been linked to many deaths due to overdose [3-7].

Artificial neural networks (ANN) are statistical learning algorithms with a flexible mathematical structure, which contributes to the identification of complex nonlinear relationships that exists between input and output data sets [8]. The mathematical model of ANNs emulates the structure and also the functionality of biological neural networks, thus being able to find models in data or to make classifications. This machine learning technique is based on a number of nodes, which are interconnected in a network-like structure and define patterns appropriate for classification purposes $[8,9]$. 


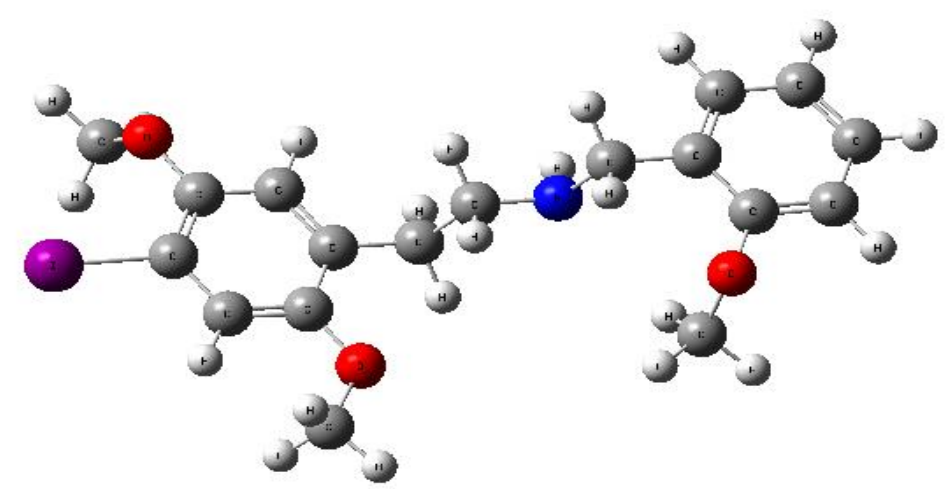

Fig. 1. Molecular structure of the 25I-NBOMe hallucinogenic phenethylamine

ANNs are trained by using algorithms based on the optimization theory and the statistical estimation theory. It calculates real gradients by using the backpropagation algorithm. In order to identify the best network architecture, the system parameters are modified in a direction related to the gradient by applying the gradient descent methodology, i.e. by determining the derivative of the cost function in relation to the network parameters [10-12].

The architecture of ANNs consists of neurons that are aggregated into layers, i.e. an input layer (formed of nodes that are the input variables), one or more hidden layers (consisting of nodes capturing the nonlinearity in the data), and an output layer (containing nodes that illustrate the dependent variables, which express the result for the given inputs).

ANNs are able to learn from experience throughout the training process and to make classifications or detect patterns. Hence, they are able to solve complex problems in situations where a large volume of data or incomplete information has to be processed [13-16]. This paper presents a series of ANNs designed to screen for NBOMe hallucinogens based on their molecular properties and charge descriptors.

\section{EXPERIMENTAL}

The development of an ANN follows two steps: building the database (i.e. collecting and preprocessing the input data) and developing the ANN model (i.e. training and validating the ANN).

In our case, the database is made up of 160 forensic substances of forensic interest, among which are drugs of abuse, precursors, and derivatized counterparts. The database was divided into 15 positives (NBOMe hallucinogens - class code NBOMe) and 145 negatives (class code non-NBOMe). The HyperChem 8.03 software package has been used in order to obtain the 3D representations of the molecular structures of all these 160 substances [17]. Then, their geometries were optimized based on the AM1 semi-empirical quantum method combined with the Polak-Ribiere mechanism. A number of 40 descriptors (14 charge descriptors and 26 molecular descriptors) were calculated for each of the 160 compounds included in the database, by using the Dragon 5.5 software package.

The first ANN was developed with all these 40 descriptors (14 charge descriptors and 26 molecular descriptors), by using the Easy NN plus software. The most important descriptors have been identified by using this network and a second ANN has been built by using only these descriptors. The architecture of both these ANNs consists of three layers, i.e. one input layer, one hidden layer, and one output layer. The training process of these ANNs was realized by using the backpropagation algorithm. After optimization, the learning rate was fixed at $\alpha=0.70$ and the momentum at $\mu=0.80$. The transfer function was the sigmoid function.

The training set of both ANNs consists of 8 NBOMe hallucinogens (see Table 1), 17 nonNBOMe compounds, and the remaining 135 samples were included in the validation set. The training process was considered complete (i.e. the convergence was reached) when the average training error (TE) falls below the target error $(\mathrm{TE}=0.01)$. The output layer of both ANNs contains two nodes (NBOMe and non-NBOMe). The cross-validation method (leave-one-out algorithm) was used to validate both ANNs. 
The first network created, named 40MP\&C-ANN, has as input variables the 40 molecular properties and charge descriptors, that have been calculated for each sample. This network has 13 hidden nodes, 546 weight connections, and reached convergence after 186 learning cycles.

The 20impMP\&C-ANN network has been built by using as input variables the first 20 most important molecular properties and charge descriptors, as determined with 40MP\&C-ANN. It has 12 hidden nodes and 264 weight connections after optimization.

\section{RESULTS AND DISCUSSION}

Table 1 presents the toxicities in mmol/L of BLTF96 (Verhaal model of Fish base-line toxicity from MLOGP), BLTA96 (Verhaal model of Algae base-line toxicity from MLOGP), and BLTD48 (Verhaar Daphnia base-line toxicity from MLOGP) of the NBOMes hallucinogens included in the training set used to build the ANNs systems.

Table 1. The values of BLTF96, BLTD48 and BLTA96 of the NBOMes hallucinogens included in the training set used to build the ANNs.

\begin{tabular}{|c|l|c|c|c|}
\hline No. & \multicolumn{1}{|c|}{ Chemical compound } & BLTF96 & BLTD48 & BLTA96 \\
\hline 1. & NBOMe 1 (25H-NBOMe) & -3.65 & -3.85 & -3.89 \\
\hline 2. & NBOMe 2 (25C-NBOMe) & -4.08 & -4.32 & -4.39 \\
\hline 3. & NBOMe 5 (25B-NBOMe) & -4.17 & -4.43 & -4.5 \\
\hline 4. & NBOMe 6 (25I-NBOMe) & -4.27 & -4.54 & -4.62 \\
\hline 5. & NBOMe 7 (25TFM-NBOMe) & -4.37 & -4.65 & -4.73 \\
\hline 6. & NBOMe 10 (25E-NBOMe) & -4.04 & -4.28 & -4.35 \\
\hline 7. & NBOMe 12 (25iP-NBOMe) & -4.23 & -4.5 & -4.57 \\
\hline 8. & NBOMe 14 (25T4-NBOMe) & -4.46 & -4.75 & -4.84 \\
\hline
\end{tabular}

The variation of the normalized error of training vs. the number of learning cycles is presented in Figure 2 for the 40MP\&C-ANN network. The relative importance, determined for the first 40 most important descriptors of the 40MP\&C-ANN system built with 40 molecular properties and charge descriptors is shown in Figure 3 in descending order.

The first 25 of 25 Example rows in descending order.

Example

T: NBOMe6

T: NBOMe5

T: NBOMe1

$\mathrm{T}: \mathrm{N} 30$

T: NBOMe7

T: NBOMe2

T: NBOMe14

T: NBOMe10

T: NBOMe12

T: N29

$\mathrm{T}: \mathrm{N86}$

T: N27

$\mathrm{T}: \mathrm{N} 23$

$\mathrm{T}: \mathrm{N} 100$

T: N64

T: N99

$\mathrm{T}: \mathrm{N54}$

$\mathrm{T}: \mathrm{N55}$

T: N11

T: N31

T: N126

$\mathrm{T}: \mathrm{N} 131$

T: N52

$\mathrm{T}: \mathrm{N14}$

T: N56
Normalized Error [0 - 1]

0.005437
0.004603
0.004540
0.003631
0.001600
0.001599
0.001473
0.001381
0.001324
0.000016
0.000016
0.000016
0.000016
0.000016
0.000016
0.000015
0.000015
0.000015
0.000015
0.000015
0.000015
0.000015
0.000015
0.000015
0.000015

Above target Below target

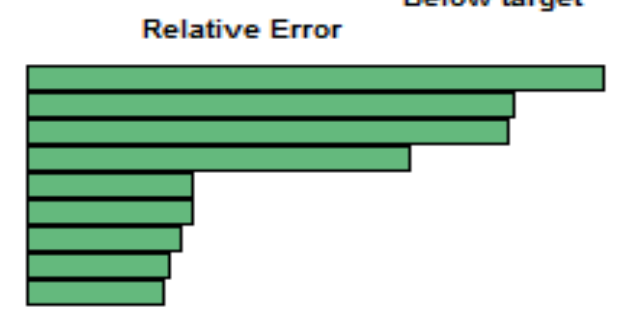

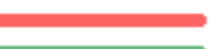

- 


$\begin{array}{lc}\text { Input Name } & \text { Importance } \\ \text { Psychotic-80 } & 45.0874 \\ \text { Depressant-80 } & 31.7842 \\ \text { TPSA(NO) } & 25.2084 \\ \text { RPCG } & 24.1057 \\ \text { TPSA(Tot) } & 23.5097 \\ \text { Psychotic-50 } & 22.4205 \\ \text { Neoplastic-50 } & 16.7783 \\ \text { Qmean } & 15.9366 \\ \text { SPP } & 15.3285 \\ \text { BLTD48 } & 15.1704 \\ \text { Hypnotic-80 } & 15.0538 \\ \text { BLTF96 } & 14.9514 \\ \text { ALOGP2 } & 14.4205 \\ \text { BLTA96 } & 14.2657 \\ \text { qpmax } & 13.7685 \\ \text { Qpos } & 12.2355 \\ \text { qnmax } & 11.8425 \\ \text { Qneg } & 11.5241 \\ \text { TE1 } & 11.1453 \\ \text { Qtot } & 11.1282 \\ \text { Ui } & 10.3698 \\ \text { GVWVAl-80 } & 10.0508 \\ \text { Infective-80 } & 10.0161 \\ \text { PCWVTe } & 9.9086 \\ \text { Q2 } & 9.1356 \\ \text { Infective-50 } & 8.9610 \\ \text { LDI } & 8.7219 \\ \text { Hypertens-80 } & 8.2483 \\ \text { RNCG } & 8.0430 \\ \text { Hypertens-50 } & 7.9127 \\ \text { MLOGP } & 7.6057 \\ \text { Depressant-50 } & 7.5228 \\ \text { ALOGP } & 7.3817 \\ \text { GVWAl-50 } & 6.9890 \\ \text { AMR } & 6.4797 \\ \text { MLOGP2 } & 5.9814 \\ \text { TE2 } & 5.9809 \\ \text { Inflammat-80 } & 4.8142 \\ \text { Hy } & 4.0491 \\ \text { Neoplastic-80 } & 3.8709\end{array}$

Relative Importance

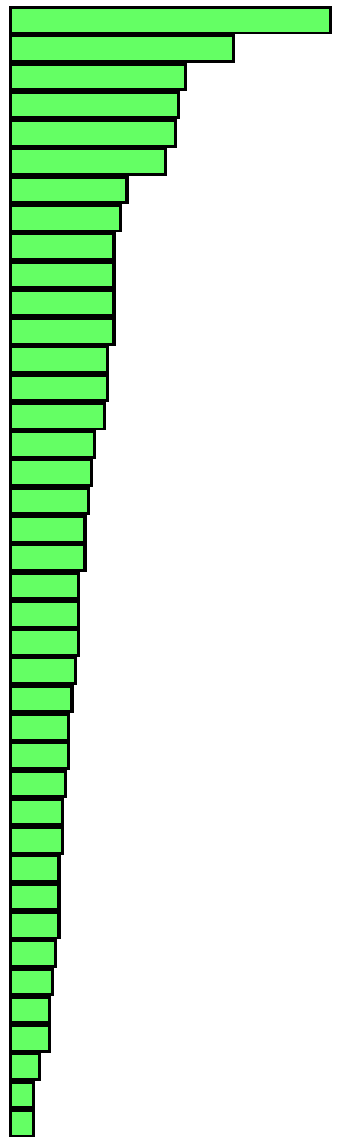

Fig. 3. The descriptors having the highest relative importance as indicated by the analysis of the $40 M P \& C-A N N$ system. Figure 5.

The training process of 20impMP\&C-ANN is presented in Figure 4 and its architecture in

20imp_Molec_prop+Charge_ANN-42cycles.tvq Learning stopped - average error below 0.0100

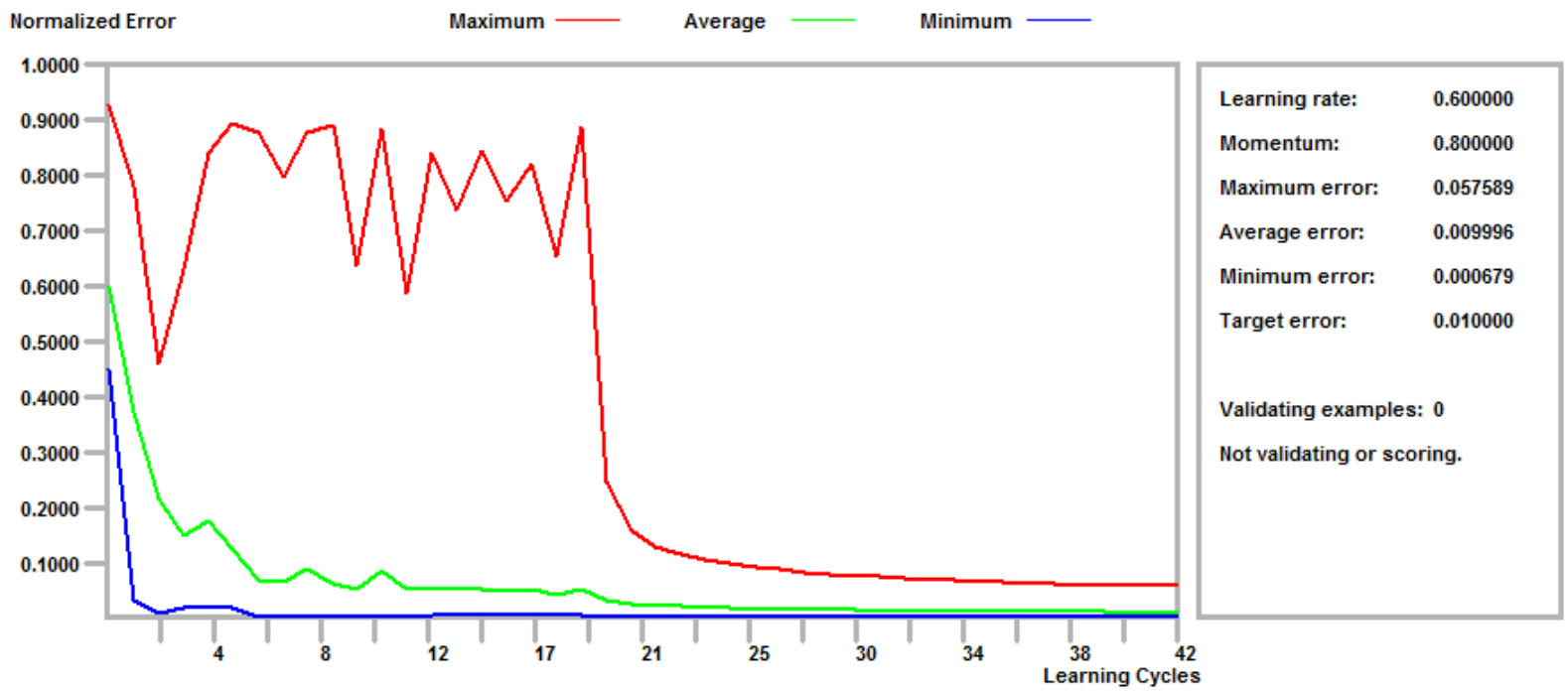

Fig. 4.The training process of the 20impMP\&C-ANN system, which was built with the first 20 most important descriptors indicated by the analysis of the 4OMP\&C-ANN network. 


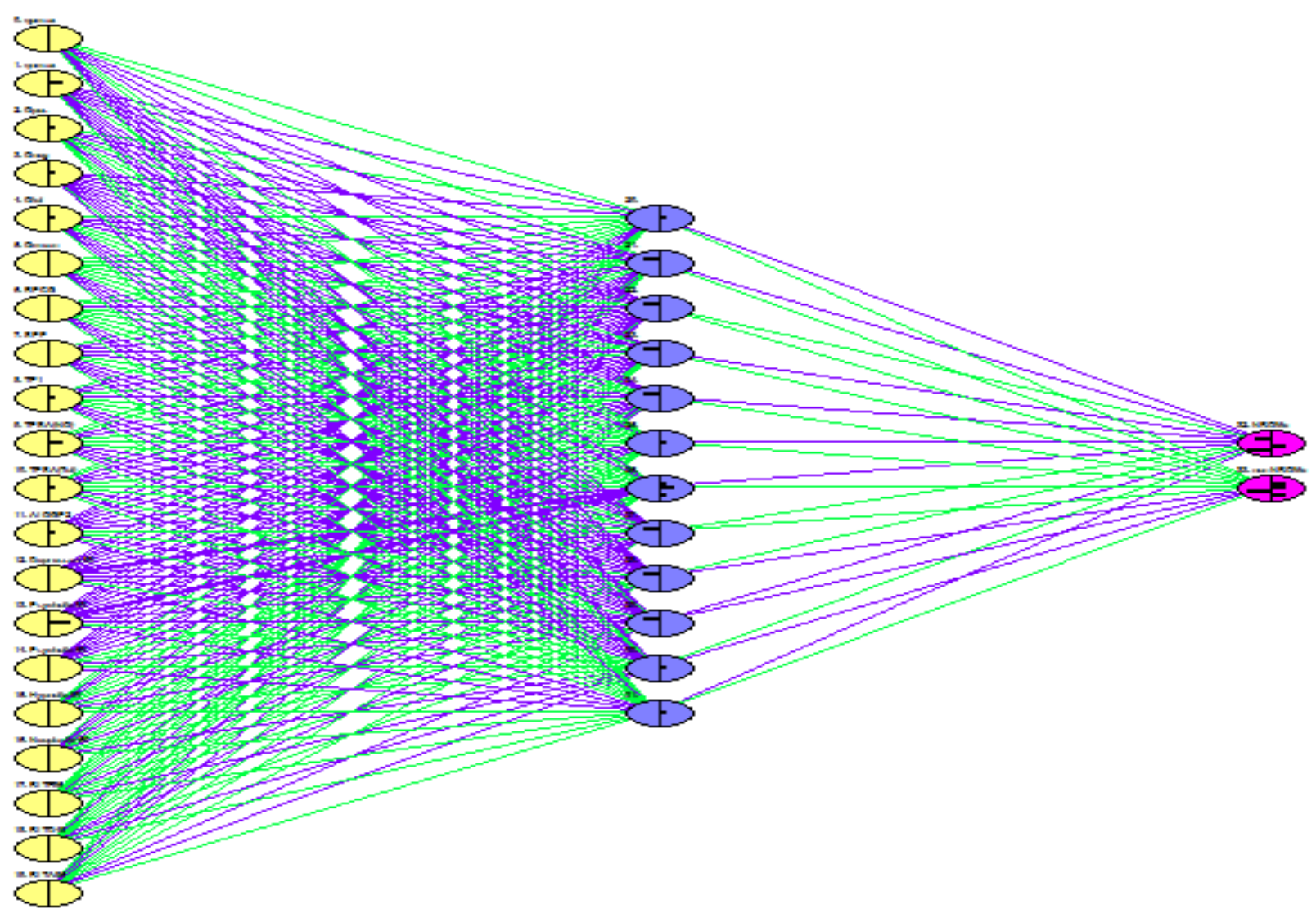

Fig. 5. The architecture of 20impMP\&C-ANN network

After validation (full cross-validation), several figures of merit were determined, in order to compare the classification efficiency of the presented ANNs: the rate of true positives (TPR), of true negatives (TNR), of false positives (FPR), of false negatives (FNR), of classification (CR), and of accuracy (ACC) (see Table 2).

Table 2. Validation of the 40MP\&C-ANN and 20impMP\&C-ANN networks.

\begin{tabular}{|c|c|c|}
\hline $\begin{array}{c}\text { Neural } \\
\text { network } \\
\text { Validation } \\
\text { parameter }\end{array}$ & 40MP\&C-ANN & 20impMP\&C-ANN \\
\hline TPR (\%) & 100 & 100 \\
\hline TNR (\%) & 87.59 & 88.97 \\
\hline FNR (\%) & 0 & 0 \\
\hline FPR (\%) & 12.41 & 11.03 \\
\hline CR (\%) & 100 & 100 \\
\hline ACC (\%) & 88.75 & 90.00 \\
\hline
\end{tabular}

Both screening systems have a remarkable sensitivity (TPR $=100 \%)$, meaning that all the positives (NBOMe hallucinogens) are correctly recognized, without exception.

Table 2 indicates that decreasing the number of variables included in the input database, based on their importance, does not affect the sensitivity of the ANNs. TPR remains equal to $100 \%$ even if the number of important descriptors is reduced from 40 to 20 descriptors. Taking into account that 
these systems are built for forensic purposes, this behavior is extremely important, as no NBOMe should be misclassified by the screening system.

The results also indicate that including in the input database only the most important molecular properties and charge descriptors improves the selectivity of the system: although the TNR remains very high for both networks, FPR is slightly lower in the case of 20impMP\&C-ANN $(\mathrm{FPR}=11.03 \%)$ than in the case of $40 \mathrm{MP} \& \mathrm{C}-\mathrm{ANN}$ ( $\mathrm{FPR}=12.41 \%)$. In addition, ACC is also slightly higher in the case of $20 \mathrm{impMP} \& \mathrm{C}-\mathrm{ANN}$.

Hence, we may conclude that 20impMP\&C-ANN is the best system that should be used for the detection of NBOMe hallucinogens.

\section{CONCLUSIONS}

Both ANN networks developed based on the most important molecular properties and charge descriptors of 40MP\&C-ANN have a remarkable sensitivity $($ TPR $=100 \%)$. Hence, all the positives (NBOMe compounds) are correctly classified. We must underline that the classification of positives is extremely important in forensic practice because the assignment of the class identity of the positives should not fail.

The 20impMP\&C-ANN network is characterized by a better selectivity (TNR $=88.97 \%)$, in comparison with the 40MP\&C-ANN network, which has a TNR=87.59\%. The analyzed networks were able to classify all the analyzed drugs $(\mathrm{CR}=100 \%)$ and also have good accuracy $(\mathrm{ACC}>$ $88.75 \%)$.

In conclusion, the 20impMP\&C-ANN system, built with the most 20 important molecular properties and charge descriptors, improves the performances of 40MP\&C-ANN. As it requires fewer input variables, the $20 \mathrm{impMP} \& \mathrm{C}$-ANN system is also requiring shorter analysis time and costs.

These systems are very useful for estimating the pharmacological activity of a new compound and eliminate the need for clinical and toxicological tests, which are costly and time-consuming.

\section{References}

1. Suzuki J., Dekker M. A., Valenti E. S., et al., Toxicities associated with NBOMe ingestion a novel class of potent hallucinogens: a review of the literature, Psychosomatics 56 (2015) 129-139.

2. https://www.deadiversion.usdoj.gov/drug_chem_info/nbome.pdf

3. Dean B. V., Stellpflug S. J., Burnett A. M., Engebretsen K. M. J., 2C or not 2C: phenethylamine designer drug review, Medical Toxicology 9 (2013) 172-178.

4. Hill S. L., Doris T., Gurung S., Katebe S., Lomas A., Dunn M., Blain P., Thomas S. H. L., Severe clinical toxicity associated with analytically confirmed recreational use of 25INBOMe: case series, Clinical Toxicology 51 (2013) 487-492.

5. Kelly A., Eisenga B., Riley B., Judge B., Case series of 25I-NBOMe exposures with laboratory confirmation, Clinical Toxicology (Philadelphia) 50 (2012) 702.

6. Suzuki J., Poklis J. L., Poklis A., My friend said it was good LSD: a suicide attempt following analytically confirmed 25I-NBOMe ingestion, Journal of Psychoactive Drugs 46 (2014) 379-382.

7. European Monitoring Centre for Drugs and Drug Addiction, EMCDDA-Europol Joint, Report on a new psychoactive substance: 25I-NBOMe (4-iodo-2,5-dimethoxy-N-(2-methoxybenzyl) phenethylamine), 2014.

8. Hsu K., Gupta H. V., Sorooshian S., Artificial Neural Network Modeling of the Rainfall-Runoff Process, Water Resource Research Journal 31-10 (1995) 2517-2530.

9. Ze W., Li X.-C., Zhu W.-X., Prediction of drug bioavailability by genetic algorithm and artificial neural network, Yao Xue Xue Bao 41 (2006) 1180-1183. 
10. Sun Y. C., Peng Y. X., Chen Y. X., Shukla A. J., Application of artificial neural networks in the design of controlled release drug delivery systems, Advanced Drug Delivery Reviews 55 (2003) 1201-1215.

11. Byvatov E., Fechner U., Sadowski J., Schneider G., Comparison of support vector machine and artificial neural network systems for drug/nondrug classification, Journal of Chemical Information and Computer Sciences 43 (2003) 1882-1889.

12. Karelson M., Dobchev D., Using artificial neural networks to predict cell-penetrating compounds, Expert Opinion of Drug Discovery 6 (2011) 783-796.

13. Gosav S., Praisler M., Dorohoi D. O., Popa G., Structure - Activity Correlations for Illicit Amphetamines Using ANN and Constitutional Descriptors, Talanta 70 (2006) 922-928.

14. Ion A., Gosav S., Praisler M., Screening for NBOMe Hallucinogens based on Artificial Neural Networks and Structural Descriptors, 2019 E-Health and Bioengineering Conference (EHB), Iasi, Romania, 2019, pp. 1-4.

15. Gosav S., Praisler M., Artificial Neural Networks Built for the Recognition of Illicit Amphetamines Using a Concatenated Database, Romanian Reports in Physics 54 (2009) 929935.

16. Gosav S., Praisler M., Dorohoi D. O., ANN Expert System Screening for Illicit Amphetamines using Molecular Descriptors, Journal of Molecular Structure 834 (2007) 188194.

17. Hyperchem software, Version 8.0.3., Hyper Co., USA, 2007. 